\title{
Combinational Services for NGN based IPTV
}

\author{
Eugen Mikóczy, Sebastian Schumann \\ NGNlab, Slovak University of Technology \\ Bratislava, Slovakia \\ \{eugen.mikoczy, sebastian.schumann\}@ngnlab.eu
}

\author{
Hans Stokking, M. Oskar van Deventer, \\ Omar A. Niamut \\ TNO \\ Delft, Netherlands \\ \{oskar.vandeventer, omar.niamut, hans.stokking\}@tno.nl
}

\begin{abstract}
This paper describes the general concept of combinational/blended services for IP-based Television (IPTV) services in next generation networks towards its service oriented architecture concept (SOA). Besides introducing general approaches of service reusability (SOA concepts), the service enablers (e.g. presence, notification, messaging) as application service function in next generation networks will be outlined by standardization work in ETSI TISPAN and also explicit examples of prototypes are given. The extensibility will be elaborated within the IPTV subsystem and also as the possible interconnection with web services. Selected cases have been successfully prototyped and deployed exemplary in the laboratory testbed.
\end{abstract}

Keywords- NGN, IPTV, services

\section{INTRODUCTION}

The concept of next generation networks (NGN) has been around for a couple of years now. Independent access, switching from vertical to horizontal design and transport layer control (i.e. QoS) are some of the key features of NGN [1]. Those new features imply a lot of advantages for the user: Device independent service access or guaranteed voice quality are only some features, that existing voice over IP infrastructures will not provide in its expected scope.

One key benefit for the network provider - actually similar to the customer's - will be the key topic of this paper: converged combinational/blended services. The actual blending concept is not regarded from access or technology point of view, but more on the service level itself.

This elaboration will mainly focus on the application plane and its content: The application service functions (ASF) and their usability and reusability within the NGN and also combinations of frameworks and subsystems respectively (e.g. integration with IP Multimedia Subsystem - IMS).

\section{NGN BASED IPTV CONCEPTS}

\section{A. NGN principles}

The changes in the NGN approach are shown in "Fig.1". This picture and many variations of it are often used to show the main idea behind the next generation concepts.
The picture shows the past and future approaches. The focus now is on where the actual services are deployed: The past required different technologies for different access networks. Each access network, technology respectively, was used to provide a particular service it has been deployed for. Services from one technology could not be used by another, a severe limitation when building blended services.

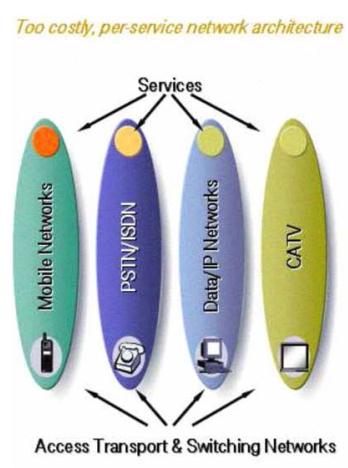

Figure 1.

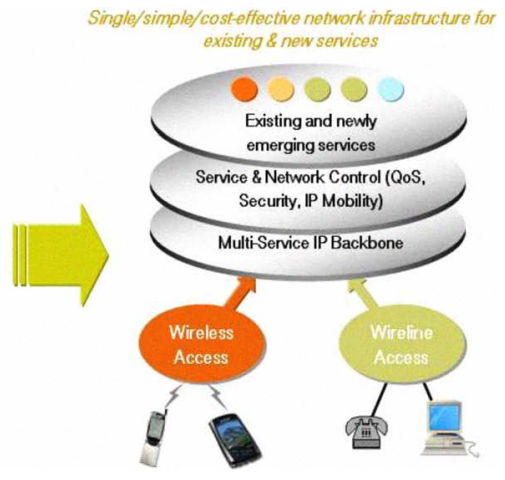

Evolution towards NGN
Considering the limitations of access devices and network capabilities, "service" as term from the past is considered to be:

- Mobile voice: Telephony

- Residential voice/ISDN voice: Telephony

- Data Access: Data processing applications, WWW

- Cable: Television

In the NGN world, each of those services will be offered by one common transport plane, providing IP access to various fixed and mobile devices. No matter how the users will finally access the network, a transport plane will make the user transparent to the higher level planes and the network: The upper control plane will see only a connection attempt from an IP endpoint. It provides the service and network control (also QoS) or performs for example authorization. The major protocol for signaling is SIP (Session Initiation Protocol). 
If the user is at some point part of the network, a third plane - the application plane - will provide the actual SIP based services to the user. Voice/media connections to other users, i.e. the replacement of the mentioned telephony-only, are provided by the control plane itself. The application plane provides all extensions to this basic feature, e.g. presence, messaging, conferencing or IP Centrex. Also, IPTV is quickly becoming a service also provided by the control plane itself, although work on IPTV services in the application plane is still in its infancy.

The messaging and presence management service will be used exemplary now, to show how services can be re-used and how next generation concepts make sense from both, customer and provider perspective.

\section{B. Service-oriented concepts in NGN}

As discussed, a network with a common application plane will be considered in the following. The old differences between the access technologies and also restrictions due to not existing compatibility are eliminated. Services can be used independent from the access technology. A service, once created, can be used multiple times.

This concept is beneficial for the provider: He can save money, deploying services only once and using them through various technologies. Furthermore, the time-to-market for new services decreases: Single services will be small and already deployed functionalities can easily be reused. Also, any implementation changes in the network will not have a big impact at the service level.

This paper will show in the following, how the presence service - originally intended for instant messaging and nowadays used also supporting telephony - will be used together with other services and by different subsystems. "SIP Presence" will be also referred to as "core service" throughout this document.

The mentioned reusability of the service follows the concept of Service Oriented Architectures (SOA): Services will be deployed in an atomic way. Each service should provide a very single function. This ensures the highest grade of reusability. A combination of more services will finally achieve the expected result. One major criterion for the mentioned reusability is well-defined interfaces for universal access by all services in the application plane. A common SOA approach is to use XML based protocols for describing those interfaces (WSDL - Web Services Description Language) and for exchanging the actual information (SOAP - Simple Object Access Protocol).

Figure 2 shows the introduced NGN planes and various ASFs in the application plane. The idea of connecting services, presence in particular, to another subsystem is to add another ASF that provides the mapping between the NGN protocol (i.e. SIP) and the protocols used in other systems.
General mapping idea behind the introduced ASF is shown on "Fig. 3". The IMS provides services within the SIP framework. If those services will be reused within other subsystems, the necessary messages and commands from the SIP world must be mapped.

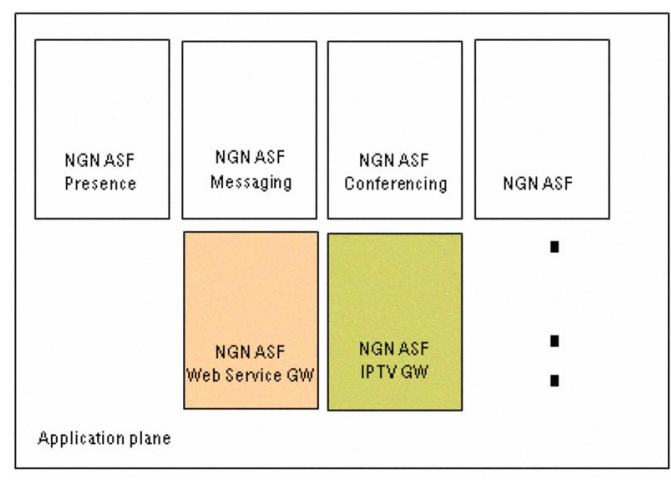

Control plane

User plane

Figure 2 .

NGN planes, focused on services

Also the messages flows and actions behind must be introduced within the new subsystem.

The picture also highlights another key aspect of Service Oriented Architectures: Services depend on their interfaces. The mapping function is clearly defined on the SIP side and on the mapped side. Whether it is the already standardized web service or the IPTV interface currently being standardized: The interfaces are important and not a specific implementation. The messages that arrive at the ASF from both sides are defined in the end. The component that processes it can be changed easily without influencing the service behavior ("Fig.3").

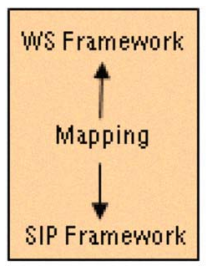

Figure 3.

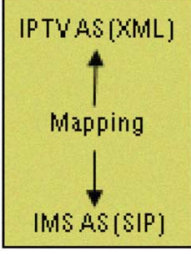

Mapping of service capabilities
The service might be scaled at some point, or its implementation changed due to various reasons. All of this has no impact on "adjacent services" as long as the interfaces are kept stable.

The following two sections will show how SIP based presence can be used within other frameworks and thus, how one service will serve various clients (being customers) as well as other network services (in our case IPTV). 
The extensions are cheap, as no extension on the core IMS side is necessary. The SIP framework provides the necessary infrastructure. The presence information, which is e.g. published from the IPTV framework or requested by the web service framework, exists in the IMS presence ASF as well as the SIP methods to perform the required actions.

The extension of the IPTV subsystem with features that the SIP presence framework provides has simple drivers:

- cheap extension (no more components or deployment costs necessary)

- quick implementation (only mapping extension required)

\section{IPTV SUBSYSTEM AND INTEGRATION WITH NGN}

Multiple standardization bodies are working on standardization of IPTV or integration of IPTV services with NGN architecture. We will focus on authors work and ideas presented in ETSI TISPAN specifications. TISPAN (Telecommunications and Internet Converged Services and Protocols for Advanced Networking) in NGN release 2 have defined NGN based IPTV architectures, specifications and protocols (work also continues with NGN Release 3).

We can list also other important activities additionally to those mentioned ETSI TISPAN IPTV specification as for example the following ones:

- Digital Video Broadcasting - Internet Protocol Infrastructure (DVB-IPI).

- ITU-T Focus Group IPTV will continue in the standardization Internet Protocol Television Global Standards Initiative (IPTV-GSI).

- Alliance for Telecommunications Industry Solutions (ATIS) initiates IPTV Interoperability Forum (IIF).

- 3rd Generation Partnership Project (3GPP) specifies MBMS (Multimedia Multicast/Broadcast Services).

- Open Mobile Alliance (OMA) proposed Mobile Broadcast Services Enabler framework.

- IETF defined most of the core protocols used in IPTV (like RTP, IGMP, MLD, RTSP, HTTP and SIP).

- Open IPTV Forum is industrial initiative with the purpose of producing end to end specifications for IPTV.

\section{A. Evolution of IPTV architectures towards NGN}

Each evolutional step realizes additional functionalities and system features to provide new value for IPTV services, for example, to increase the Quality of Experience (QoE) for end users and to converge TV with other telecommunications and interactive multimedia services. A quick and easy introduction of new service features and reduction of the operating costs may be another important motivation to evolve the IPTV systems (as shown in Fig.4) [2].

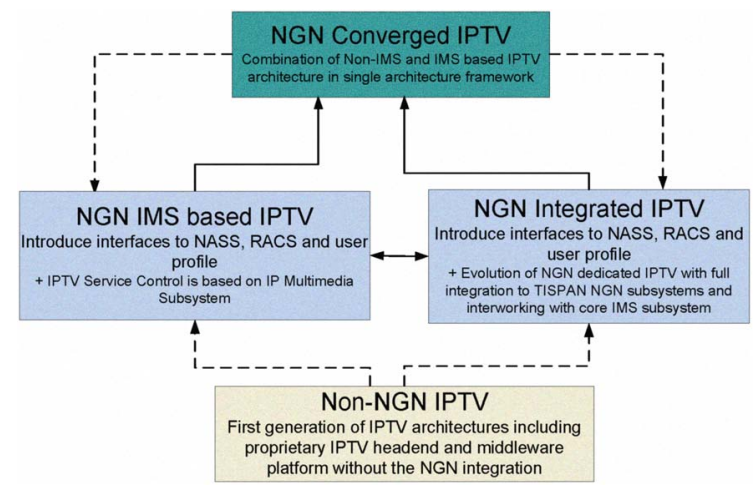

Figure 4. The potential IPTV migration paths and evolution steps for NGN based IPTV architectures.

In comparison with proprietary non-NGN IPTV solutions, an NGN dedicated IPTV system can provide standardized IPTV services and features with standardized IPTV control and media delivery functions. The NGN based IPTV in both cases enables the integration with User Profile Server Function (UPSF) and with transport control subsystems called Network Attachment Subsystem (NASS) and Resource and Admission Control Subsystem (RACS). NGN based IPTV concept allows to realize personalized value-added IPTV features and to use existing NGN network resources more efficiently.

The evolution to NGN IMS based IPTV is based on the observation that IMS acts as a unified service control platform for all future NGN services, therefore also for IPTV. NGN based IPTV can be therefore inherently integrated (in case of IMS based IPTV) or only interwork (in case of NGN integrated IPTV) with the NGN IMS based service platforms.

On the other side, however, we cannot expect all NGN services in the future will be only IMS based. So convergence and combination/interaction of IMS with non-IMS IPTV to be realized as NGN converged IPTV, this can be foreseen in the future common architecture framework (NGN converged IPTV).

\section{B. COMPARISON OF NON-IMS AND IMS BASED NGN IPTV ARCHITECTURES}

In the ETSI TISPAN NGN R2, several stage 1 (requirements) and stage 2 (architecture) specifications are addressing the IPTV integration within TISPAN NGN standards:

- IPTV service requirements [4]

- $\quad$ Dedicated IPTV subsystem architecture [5]

- $\quad$ IMS support for IPTV architecture [6]

The specifications about implementation of IPTV functions, interfaces, procedures, protocol recommendations of stage 3 have been finalized and more information can be found for NGN dedicated IPTV in ETSI TS 183064 [7] or for IMS based IPTV in ETSI TS 183063 [8]. 
TABLE I. POSSIBLE MIGRATION AND SWITCH OVER SCENARIOS:

\begin{tabular}{|c|c|c|c|c|c|}
\hline $\begin{array}{c}\text { Evolution } \\
\text { step }\end{array}$ & $\overline{U E}$ & Transport & $\begin{array}{c}\text { Media } \\
\text { control } \\
\& \\
\text { delivery }\end{array}$ & $\begin{array}{l}\text { Service } \\
\text { control }\end{array}$ & Application \\
\hline Non-NGN & STB & $\begin{array}{l}\text { Content } \\
\text { network }\end{array}$ & delivery & \multicolumn{2}{|c|}{ IPTV middleware } \\
\hline $\begin{array}{l}\text { TISPAN } \\
\text { NGN } \\
\text { integrated } \\
\text { IPTV }\end{array}$ & $\overline{\mathrm{UE}}$ & $\begin{array}{l}\text { Transport } \\
\text { processin } \\
\text { g, NASS } \\
\text { \& RACS }\end{array}$ & $\begin{array}{l}\text { MCF \& } \\
\text { MDF }\end{array}$ & $\begin{array}{l}\text { IPTV-C } \\
\text { Interaction } \\
\text { with IMS } \\
\text { and other } \\
\text { NGN } \\
\text { subsystems }\end{array}$ & CFIA, SD\&S \\
\hline $\begin{array}{l}\text { TISPAN } \\
\text { IMS based } \\
\text { IPTV }\end{array}$ & $\overline{\mathrm{UE}}$ & $\begin{array}{l}\text { Transport } \\
\text { processin } \\
\text { g, NASS } \\
\text { \& RACS }\end{array}$ & $\begin{array}{l}\text { MCF \& } \\
\text { MDF }\end{array}$ & IMS & $\begin{array}{l}\text { SCF, } \\
\text { SDF }\end{array}$ \\
\hline $\begin{array}{l}\text { NGN } \\
\text { converged } \\
\text { IPTV * }\end{array}$ & $\overline{\mathrm{UE}}$ & $\begin{array}{l}\text { Transport } \\
\text { processin } \\
\text { g, NASS } \\
\text { \& RACS }\end{array}$ & $\begin{array}{l}\text { MCF \& } \\
\text { MDF }\end{array}$ & \multicolumn{2}{|c|}{$\begin{array}{l}\text { *This part is for future study } \\
\text { in case of TISPAN NGN } \\
\text { IPTV architectures }\end{array}$} \\
\hline
\end{tabular}

\section{NGN integrated IPTV architecture}

The NGN integrated IPTV subsystem as shown in "Fig.5" provides basic integration of IPTV functions to NGN architecture and other NGN subsystems especially with UPSF, and transport control RACS and NASS [5].

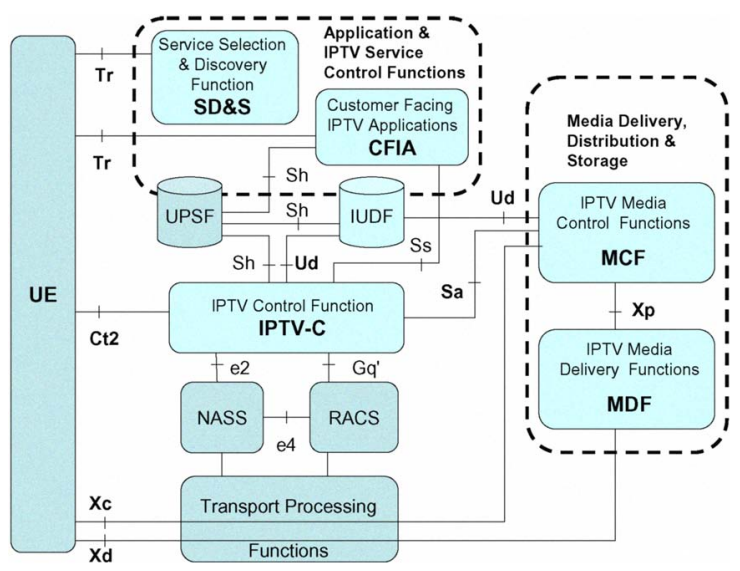

Figure 5.

Simplified TISPAN NGN integrated IPTV subsystem functional architecture [5].

A user can with his user equipment (UE like set-topbox STB) access the service description via SD\&S service, selection and discovery procedures follow DVB IPI specification and use http protocol over the $\operatorname{Tr}$ reference point. The same $\mathrm{Tr}$ interface can be used by UE for accessing the user interface and service selection over Customer Facing IPTV application (CFIA). CFIA provides over the Tr http based interface IPTV service provisioning, selection and authorization. IPTV control (IPTV-C) is enabled over the $\mathrm{Ct} 2$ interface (http or RTSP control). Media (e.g. content on demand - CoD) can be streamed by unicast or multicast over the $\mathrm{Xd}$ interface from Media Delivery Function (MDF) and control via the Xc interface by Media Control Function (MCF). UE can also access common services in NGN via interactions with NGN applications.

\section{NGN IMS based IPTV architecture}

The second alternative and next evolution step is replacing of the IPTV control by IMS based subsystems (based on [6], [9]) and introduction of session based IPTV service control based on SIP protocol "Fig.6".

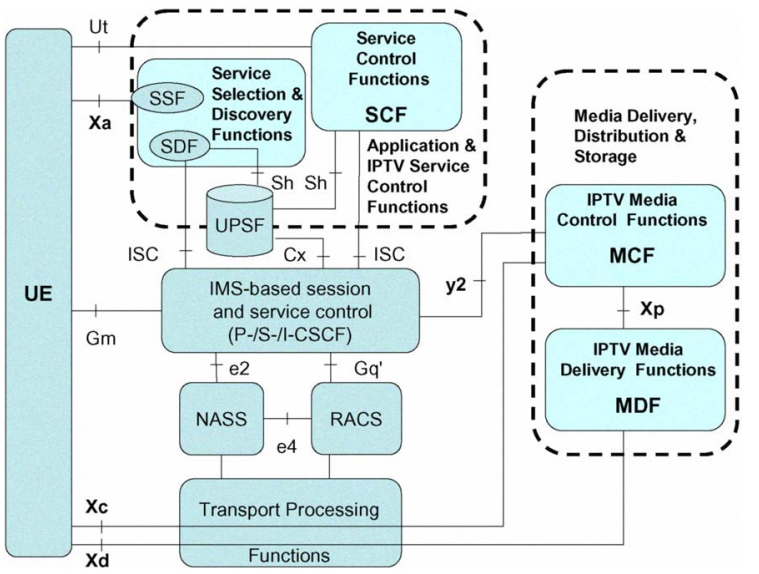

Figure 6. Simplified TISPAN NGN IMS based IPTV functional architecture [6]

The service discovery is performed by SIP application servers called the Service Discovery Function (SDF). The SDF provides information about the Service Selection Function (SSF). The SSF contains necessary service information (e.g. TV programme guides) for initiating IPTV services. The Service Control Function (SCF) is responsible to serve SIP based service initiation requests delivered over $\mathrm{Gm}$ interface via IMS core for any IPTV services required from the UE. The MCF and MDF functionalities are similar to those in NGN dedicated IPTV with difference that SIP requests can be forwarded from SCF to MCF via IMS core and y2 interface. The resource allocation and reservation is performed over existing IMS interface to the RACS (Gq'). Specific combinations of SIP and RTSP protocols are designed for CoD service initiation where a SIP INVITE message is used for session setup but control of the media stream is handled via Xc using RTSP.

The IMS based IPTV system has a number of advantages because IMS can act as unified service control subsystem for all NGN services instead of establishing an additional specialized subsystem (as is the case with an NGN integrated IPTV subsystem). Additionally IMS can more naturally support mobility, interaction with NGN service 
enablers (like messaging or presence), service personalization or quadruple play services (voice, data, video and mobile) [2].

\section{EXPERIENCES WITH SOA BASED APPLICATION FRAMEWORKS FOR NGN BASED IPTV}

Another advantage of applying service oriented concepts to the NGN is the ability to change the implementation of a service, without changing the interface. In current migrations from one telecommunications system to another, not only do interfaces change but the new system consists of a whole new implementation. Migrations are thus often so-called complete migrations: getting rid of a complete old system (e.g. IN) and introducing a complete new system (e.g. IMS). One of the key concepts of Service Oriented Architectures is the ability to loosely couple the service and the service users. The service implementation can e.g. first be based on a legacy system with a wrapper providing a web service interface, while later on the service implementation can be changed at will.

Introducing such interfaces, e.g. Parlay and Parlay-X, in telecommunication can also provide these kinds of benefits. Applying this to the IPTV subsystem, one can clearly see certain advantages. One of this is in the introduction of a socalled network-initiated media control.

The main approach for handling service interaction in a next generation network is by introducing a central service interaction manager, like the Service Capability Interaction Manager in IMS. This functionality, sometimes also called a Service Broker, is a centralized module through which all commands can go, so it can control the feature interaction. With standard telephone services, this usually consists of socalled service orchestration, i.e. deciding which services are applicable to a certain call, in which order, and even handling dependencies, e.g. only run service B if service A returns a certain outcome.

For IPTV services and blended services such a centralized interaction manager is still for further study. First of all, there are different options for implementing such a service broker in IPTV architecture. This is not easy, since the module should receive all commands given, which include all actions initiated by a user with their remote control. All centralized solutions have this disadvantage: it usually does not scale well, and if too many different signals have to be processed, it is also hard to configure everything centrally.

Another consideration for placing an interaction manager in the IPTV architecture is if services by different parties are expected. If the Internet way of providing services also applies to IPTV, a lot of different service providers can be expected to want to deliver services through the same IPTV architecture. In Next Generation Networks other parties, also called third parties, can gain access to an infrastructure through the use of third party service interfaces like Parlay. This will make the issue of service interaction more challenging, because the IPTV provider can not know the details of every service supplied, and thus cannot decide on e.g. service precedence.

\section{A. Web services based application framework}

The drivers for the web service extension are similar to the ones for IPTV. In addition, the web service framework has already well-defined API's for many service functions presence or messaging being ones of them.

In general, all mentioned APIs are summarized by the ParlayX framework [10]. It is a less-complex variant of the Parlay APIs.

It can contain a set of telecommunication web services, including presence, messaging, call notification, etc. Each specification provides

- namespaces

- sequence diagrams

- data definitions

- interface specifications and detailed method descriptions

- fault definitions

- service policies

- WSDL interface description.

The main problem is that the web service will have to interact with the SIP framework. The interface towards the web service clients is defined, but messages and the exchanged information towards the SIP framework must be mapped.

Currently, there already exist some recommendations for this mapping. According to the different approaches in SIP and web services, this problem had to be elaborated further.

The prototype of ParlayX web service for presence for example implements three interfaces:

- PresenceConsumer: watcher methods

- PresenceSupplier: presentity methods

- PresenceNotification : watcher notification interface for • presence events

The first two interfaces have to be implemented to allow retrieval and publication of presence states. The deployed gateway with those functions can already serve for all major methods:

- Subscription to presence state (fetching or polling mode)

- Publication of presence state

- Watcher-information (pending, active, terminate watcher) and modification

The SIP methods implied by this web service interaction have been well-defined in the NGNlab testing laboratory (extending [11]) and proven to work.

The attribute mapping was a major challenge in this, as the SIP world (PIDF, RPID) defines different presence attributes than the web service world defined by ParlayX.

The following part of paper is presented two different examples of IMS based IPTV architectures to clarify how service interaction can be achieved by using this existing work of relevant standardization bodies (e.g. from OSA/Parlay or 3GPP). For these two possible architectures, it is assumed that 
service interaction requires a dedicated Application Server that may explicitly implement the blended service functionality (e.g. pausing the TV on incoming call, or watching apart together, presence, etc.). Such an AS can be seen as an external application with respect to the TISPAN IPTV architecture.

First approach is based an OSA/Parlay/Parlay X Service Capability Server (SCS), that provisions standardized interfaces and APIs to external and third party application servers. The OSA SCS acts as a secure gateway between the underlying network and the application with the OSA architecture. It is responsible for providing specific service abilities to third party applications ("Fig.7").

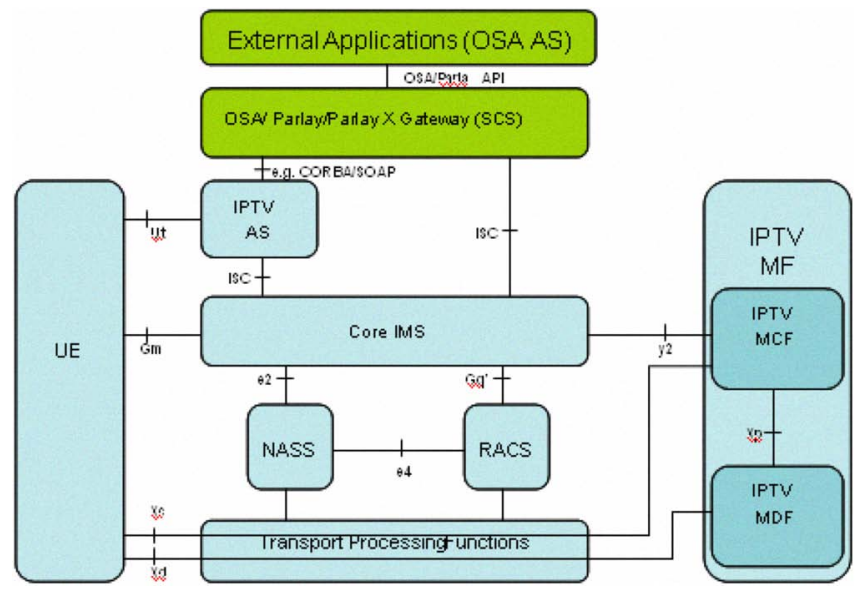

Figure 7. Interaction between TISPAN IMS-based IPTV architecture and an OSA/Parlay/Parlay X SCS. [12].

An SCS further serves to make an abstraction of the functionality offered by the network, in effect offering the service capability features of the underlying network to the third party applications.

Second approach for IMS based IPTV (shown "Fig.8") is based on a Service Capability Interaction Manager (SCIM) that allows for service association and dynamic service triggering.

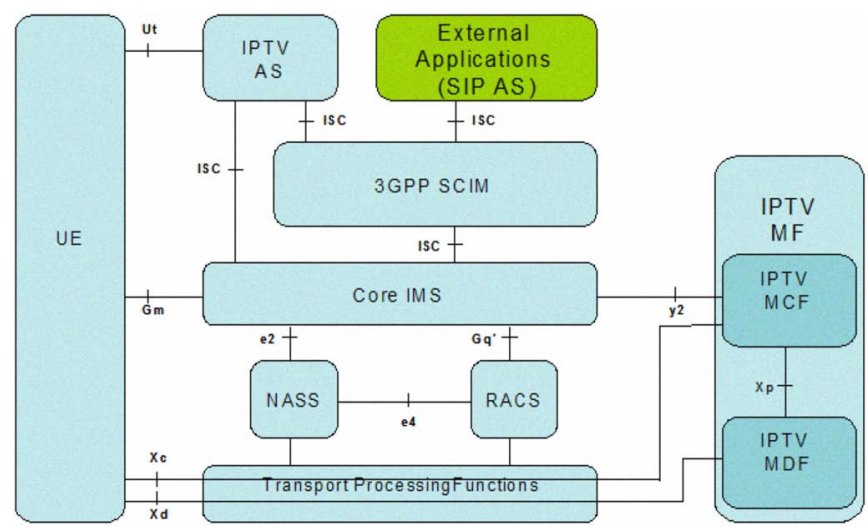

Figure 8. Interaction between TISPAN IMS-based IPTV architecture and a 3GPP SCIM
SCIM is a function that manages the interaction between IPTV services and other NGN services, by e.g. associating the IPTV services with other NGN services and dynamic triggering the services in the ongoing session based on the information about the relational session or user's status. Both approaches adopt a separation of the application plane from the control and transport plane, thereby allowing blended services. Both the OSA AS in the first approach and the SIP AS in the second approach, are well equipped to not only connect to the IPTV control plane but also to the voice services (telephony) domain. In this way, blended services can be implemented straightforwardly.

\section{B. Application in NGNlab testbed for NGN based IPTV (non IMS \& IMS)}

The NGNlab testbed contains all necessary functions to demonstrate the possible NGN and IPTV interaction within a proof of concept. The existing IPTV AS and its respective clients have been extended with the required SIP components (i.e. UE, SIP Registrar/Proxies in NGN, in the future IMS UE/IFC in IMS S-CSCF) and the mapping ASF. "Fig. 9" shows that actions on the side of user A imply actions in both, user B SIP UE and IPTV UE/STB.

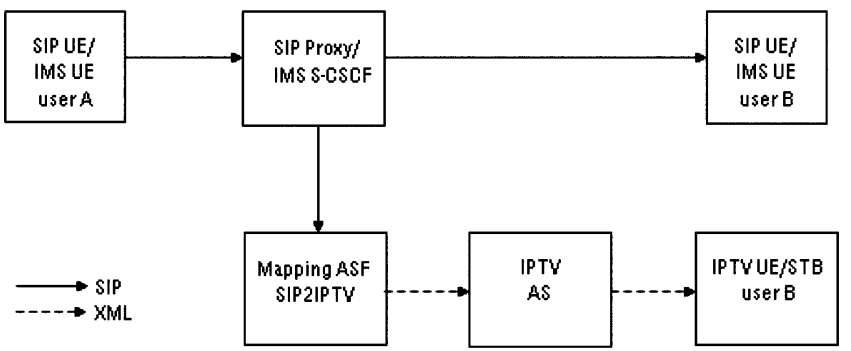

Figure 9. Test platform for IPTV interaction

The SIP UEs are normal clients that register with the SIP registrar. If user A wants to interact with user $B$, it is required to send all SIP messages through the SIP proxy. The testbed uses SIP INVITE messages for call creation and SIP MESSAGE for text interaction. As the SIP proxy is always inthe-middle of the user interaction, it was the obvious point to connect to the mapping ASF. The SIP proxy will fork calls to the mapping ASF and forward also the SIP MESSAGE messages there.

The mapping ASF is a self-deployed small application, which performs the necessary tasks based on a similar concept of common SIP2IPTV gateway located in NGN ASF as was presented in ETSI TISPAN and standardized in NGN dedicated IPTV stage 3 specification [7] in Annexes A.5 (messaging), A.6 (presence services), A.7 (IPTV service interaction with NGN/IMS services e.g. incoming calls), A.8 (Notification).

The common NGN ASF is responsible to terminate and process multiple incoming messages (i.e. SIP, MM7, SMTP, other) into generic XML schemas (using same IPTV 
notification framework), which are then passed to CFIA for presentation to the UE. The responses from the UE via CFIA should be passed back to the common NGN ASF, which will route the messages to the appropriate destination.

So far, the practical tests were directed also towards the existing non NGN IPTV AS (actually unidirectional way only). Future test cases will also allow actions from the IPTV (e.g. initiate a call, subscribe presence state of a user), responses to messages (e.g. reject an incoming call) and will be integrated with the IMS testbed in NGNlab.

The above shown examples can be easily applied to the IMS environment as well: The SIP proxy could be understood as specialized IMS application server. The mapping ASF on the application plane will communicate with other ASF (e.g. presence server) or directly with the control plane for basic scenario interactions (e.g. calls). The interaction with the control plane can be triggered at the S-CSCF. Setting up IFC (Initial Filter Criteria) the S-CSCF can determine which messages it has to forward to the ASF under which conditions. IFCs can be set for general conditions (acc. SIP message criterions), but also user specific. In the latter case, they are stored in the HSS as part of the user profile.

\section{Applications in TNO testbed for NGN based IPTV (using IMS)}

Consider the use case of TNO prototype of IPTV blended service called 'pause-tv-on-incoming-call'. In this use case, the user's video stream is paused when an incoming call is answered. For broadcast TV this pausing means recording the stream for later viewing, also called delay-TV. Some existing non-NGN solutions already provide several implementations (usually proprietary development per solution vendor) of such a feature but prototyping such a scenario by standardized technology based on IMS based service orchestration and web services enable easier and faster implementation as well as higher flexibility to modified service scenarios for the future use cases. Recording itself can e.g. be implemented on a user's set top box, if this set top box contains a harddisk and recording capabilities. Local recording may be an easy implementation to launch such a service, because little investments are necessary and no discussion arises on digital rights. But recording could also be done inside the network, i.e. using a network PVR. Although there are ongoing discussions about if this is allowed [13], it clearly is technically efficient if more then one user chooses to record a certain program. Using a service oriented implementation offering a generic interface capability, migration of one of these implementations to the other and even co-existence of these implementations will have only little impact on the overall service. But, since the horizontal layering of services above several networks offers new service opportunities, new service interaction opportunities also arise. Given the use case of 'pause-tv-onincoming-call' which we call FoneFreez, the question is how this will work when users can also manually pause the TV, using their remote control. Suppose someone uses the remote to pause the TV, e.g. to go outside to get a bite to eat. But, what happens if this household receives a phone call and someone else picks up the phone? Normally, ending the conversation would cause the TV to start playing again, but that clearly is not what should happen right now. Also consider another case, where the person receives a phone call but wants to continue watching the television program. In this case, the instructions from the remote control must also supersede the instructions from elsewhere, but how is the network to know this? Handling such interactions requires proper state model and information about the actual service status to be part of the interfaces from the control plane to the application plane.

As "Fig.10" clearly shows, combinational services not only use telephone services for which such standardized interfaces exist, also other core services in the control plane such as IPTV services have to offer standardized interfaces to the application plane.

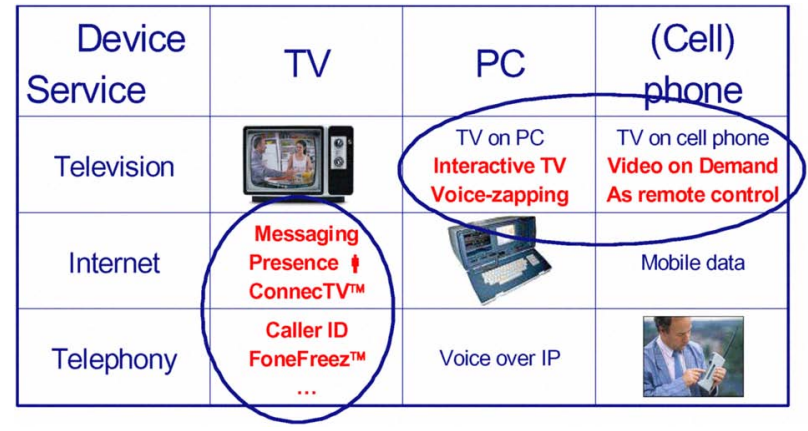

Figure 10. New Service Opportunities for blended services

In the lab at TNO we implemented the 'pause-tv-onincoming-call', using our IMS-testbed. To implement this service, we developed a control interface for TV media actions, such as changing channels and pausing and playing a TV stream. In the IMS application server we implemented a database, correlating the phone numbers to the User Equipment used for TV playback. ("Fig.11").

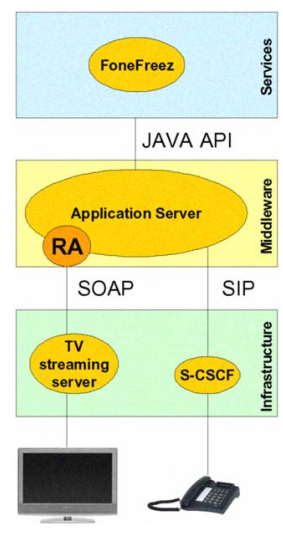

Figure 11. The TNO FoneFreez Implementation

We first implemented the pausing through the use of VLC [14], which offers timeshifting capabilities. We streamed the output of the VLC across the network to a settop box using multicast, simulating an IPTV environment. But, we also implemented the pausing through the use of a recordingcapable settop box, containing a harddisk for recording media. 
Even though the implementation of the service (pausing the TV) changed completely, it did not change the interface specified for use by the IMS application server! This clearly shows the strength of the separation of the application plane from the control plane. Because such new combinational services have to work with existing networks and existing NGN systems, good interfaces play a very important role here. If these combinational services are to be part of the application plane, only loosely coupled to the control plane via standardized interfaces, all core services delivered in the control plane must be offering such interfaces.

\section{Future work}

TISPAN NGN release 2 introduce mainly existing type of services (broadcast, content on demand, network personal video recording) and just minimally show interaction between services. Release 3 in IPTV specification already include some new features and services like user recommendation, targeted advertising, user generated content, etc. Extended group of services required more sophisticated interaction possibility with existing services and their service enablers.

New issues related to IPTV also mean increasing demand to have a testbed to be able prototype and test different concepts. The team of authors has been working on several topics related to personalization, service blending, content distribution, intra/inter domain interconnection, mobility and roaming issues which are most challenging topics for near future of NGN based IPTV.

\section{CONCLUSION}

Service blending and interaction or integration between IPTV and NGN can be achieved by different approaches presented in the paper. The first simple step could be made with a simplified interworking applicable also for existing NGN solution and non-NGN solution via a SIP2IPTV gateway. In a next evolution step this GW can become an IPTV ASF factional element described in NGN integrated IPTV solution. The most advanced solution is provided by implementation of IMS based IPTV with described interaction framework [12] reusing existing OSA/Parlay SCS Gateway or SCIM concepts.

Presented testbed and experience with prototyping specific use cases of combinational services show the need to solve also specific issues related to service/user/UE specific behavior in case of integrating different systems/architectures or service enablers. Core services can be accessed through various ways by the customer (mobile applications, phones, web front-end, via web services) or the operator himself (gateways that map interfaces, directly using SIP) or $3^{\text {rd }}$ parties. Therefore unified interfaces and reusing of SOA principles will enable easier integration and service development.

\section{ACKNOWLEDGMENT}

The authors have been actively participating on ETSI TISPAN NGN Release 2 and release standardization with several agreed contributions included in TISPAN NGN R2/R3 specifications [15] (e.g. WI0005, WI2048, WI2049, WI3127, WI3137, WI7029, WI1059, WI2070, WI2074). Some parts of this paper are based on ETSI TISPAN contribution of authors.

This paper also presents some of the results and acquired experience from various research project such as NGNlab project [16], European Celtic-EURECA project Netlab [17], Slovak National research projects: AV project 4/0019/07: Converged technologies for next generation networks (NGN), Slovak National basic research projects VEGA 1/0720/09 and VEGA 1/4084/07.

This paper has been partly supported by SenterNovem, an agency of the Dutch Ministry of Economic Affairs. The TNO part of its writing has been carried out in the context of the European EUREKA CELTIC RUBENS project.

\section{REFERENCES}

[1] ETSI ES 282001 V1.1.1 (2005-08), TISPAN; NGN Functional Architecture Release 1, 2005

[2] Mikoczy, E., Sivchenko, D., Xu, B., Moreno, J.: "IPTV services over IMS - Architecture and standardization", in IEEE Communication Magazine, Vol.47, No.5, May 2008, p. 128, ISSN: 0163-6804, 2008.

[3] Mikóczy, E.: “ Next Generation of Multimedia Services - NGN based IPTV architecture“, In: 15th International Conference on systems, Signals and Image Processing IWSSIP 2008, 25 - 28 June, 2008, Bratislava, Slovak Republic.

[4] ETSI TS 181016 V2.0.0 (2007-11), TISPAN; Service Layer Requirements to Integrate NGN Services and IPTV, 2007

[5] ETSI TS 182028 V2.0.0 (2008-01), TISPAN; IPTV Architecture; Dedicated subsystem for IPTV functions, 2008

[6] ETSI TS 182027 V2.0.0 (2008-02), TISPAN; IPTV Architecture; IPTV functions supported by the IMS subsystem, 2008

[7] ETSI TS 183064 V0.1.1 (2008-06), TISPAN; Dedicated IPTV subsystem Stage 3 Specification, Technical Specification Draft, 2008

[8] ETSI TS 183063 V2.0.2 (2008-04), TISPAN; IMS based IPTV Stage 3 Specification, 2008

[9] 3GPP TS 23.228 V7.7.0 (2007-03), Technical Specification, IP Multimedia Subsystem (IMS); Stage 2, 2007

[10] ETSI TISPAN ES 202 391-14 v1.2.1 ParlayX web services; Part 14: Presence

[11] ETSI TISPAN TR 102 397-14-2 v1.1.1 Mapping of Parlay X Web Services to Parlay/OSA APIs; Part 14: Presence Mapping; Sub-part 2: Mapping to SIP/IMS Networks

[12] ETSI DTS 182027 V3.0.3 (2008-10), TISPAN; IPTV Architecture; IPTV functions supported by the IMS subsystem, Technical Specification Draft, 2008

[13] http://www.lightreading.com/document.asp?site=cdn\&doc_id=160765

[14] Video LAN Client software, open source code available at http://www.videolan.org/

[15] ETSI TISPAN portal, http://www.etsi.org/tispan/

[16] NGNlab - NGN laboratory at Slovak University of Technology in Bratislava, http://www.ngnlab.eu

[17] NetLab: Use Cases for Interconnected Testbeds and Living Labs, http://www.celtic-nitiative.org/Projects/NETLAB/default.asp 